\section{Ichthyofaunal diversity of two beels of Goalpara District, Assam, India}

\section{Susmita Saha ${ }^{1} \&$ Sabitry Bordoloi ${ }^{2}$}

\author{
${ }^{1}$ Dalgoma Higher Secondary School, Dalgoma, Goalpara, Assam \\ 783125, India \\ ${ }^{2}$ Resource Management and Environment Division, Institute of Advanced \\ Study in Science and Technology, Paschim Boragaon, Guwahati, Assam \\ 781035, India \\ Email: ${ }^{2}$ sabitrybordoloi@ rediffmail.com
}

In Assam, floodplain wetlands are known as beels. Beels are highly productive ecosystems that contribute to the fishery resources of the state, and their socio-economic, commercial and ecological values are well established. The present study is based on a collection of fish (2005-2007) from Sidli Beel and Seksekia Beel of Goalpara District of lower Assam (90 96'$\left.91^{\circ} \mathrm{O} 5^{\prime} \mathrm{E} \& 25^{\circ} 24^{\prime}-26^{\circ} 54^{\prime} \mathrm{N}\right)$. This is the first survey of ichthyofaunal diversity in these beels, upon which the people of neighbouring villages depend for their livelihood.

The state of Assam has the distinction of possessing a large number of floodplain wetlands (Numbers $=3513$, Area $=$ 0.101 million ha) (ARSAC 1997), which account for nearly $50 \%$ of the total floodplain wetland area (0.205 million ha) in India. In terms of potential, the beels of Assam are capable of producing 50,000 tons of fish per year (Dutta \& Lahon 1987). Dey $(1981,1984)$ studied the hydrobiology and productivity of some commercially important beels of Kamrup district of Assam. Lahon (1983) studied the limnology and fisheries of some commercial beels of Assam. Yadav (1987) and Yadav et al. $(1981,1986,1987)$ studied the various fishing methods used in beels. Das \& Bordoloi (1997) recorded fish fauna in different ecological zones in the Basistha River, Kamrup District, Assam which enrich the fish fauna of Deepar beel, Guwahati. Goswami et al. (1999) studied eutrophication stresses in some wetlands of Assam. Sen (2003) has summarised the threatened and endemic fishes of northeastern India. Bordoloi \& Baishya (2006) recorded a fish Puntius ornatus from the beels in Hajo, Kamrup District, Assam that is a new record for the Brahmaputra drainage

\section{Description of the study area}

The fish fauna of different segments of the Brahmaputra

Date of publication 26 April 2009

ISSN 0974-7907 (online) | 0974-7893 (print)

Editor: W. Vishwanath

Manuscript details:

Ms \# 01806

Received 11 June 2007

Final revised received 16 December 2007

Finally accepted 21 February 2008

Citation: Saha, S. \& S. Bordoloi (2009). Ichthyofaunal diversity of two beels of Goalpara District, Assam, India. Journal of Threatened Taxa 1(4): 240-242.

Copyright: (C) S. Saha \& S. Bordoloi 2009. Creative Commons Attribution 3.0 Unported License. JoTT allows unrestricted use of this article in any medium for non-profit purposes, reproduction and distribution by providing adequate credit to the authors and the source of publication.

\section{OPEN ACGESS | FREE DOWNLOAD}

river varies depending upon the feeding tributaries. Sidli and Seksekia beels are situated on the south bank of the river. The two beels are connected by a channel, and Sidli Beel is connected to the river via Solmari channel. Before the great earthquake of 1950 the water level in these beels was maintained year round, but now the water level lowers during the winter season in the opinion of villagers, and connection with the Brahmaputra is maintained only during the monsoon. The Seksekia Beel has two parts; the southern part is known as Bigger Seksekia and the northern as Smaller Seksekia.

Morphometry of Sidli Beel

Maximum effective length Maximum effective depth

Maximum width

Mean depth

$2.0 \mathrm{~km}$ (approx.)

$1.5 \mathrm{~m}$

$37.0 \mathrm{~m}$

$1.4 \mathrm{~m}$

Morphometry of Bigger Seksekia Beel

Maximum effective length $0.5 \mathrm{~km}$ (approx.)

Maximum effective depth $1.5 \mathrm{~m}$

Maximum width $38.0 \mathrm{~m}$

Mean depth $1.2 \mathrm{~m}$

Morphometry of Smaller Seksekia Beel

Maximum effective length $0.75 \mathrm{~km}$ (approx.)

Maximum effective depth $1.0 \mathrm{~m}$

Maximum width 30.0m

Mean depth $0.8 \mathrm{~m}$

\section{Materials and Methods}

Diversity of fish fauna was monitored from 2005-2007 in Sidli and Seksekia beels. Recording was done at fortnightly intervals. Common fishes were recorded in the landing site, and a sample collection was made for certain species for further laboratory confirmation. Landing sites were monitored at weekly intervals for a period of two years, covering premonsoon, monsoon and post-monsoon seasons. For identification and classifications, Talwar \& Jhingran (1991) and Jayaram (1999) were followed, while nomenclature was based on Fishbase (2007). Important publications on fishes of the northeastern region of India by Sarkar \& Ponniah (2000) and Sen (2003) were referred to. Specimens were preserved in $6 \%$ formaldehyde solution.

\section{Results and Discussions}

Table 1 presents a list of all the fish recorded from Sidli and Seksekia beels. Scientific name, local name and conservation status as per Freshwater Fish CAMP of India (Molur \& Walker 1998) are compiled. Altogether, 59 species belonging to 40 genera, 19 families and eight orders have been recorded to date. A total of 17 riverine fishes were recorded, the rest are commonly found in lentic habitats. Fish from lotic habitats were: Gudusia chapra, Salmophasia bacaila, Barilius bendelisis, Cirrhinus reba, Catla catla, Labeo calbasu, Labeo bata, Labeo gonius, Labeo rohita, Wallago attu, Ompok pabda, Ailia coila, Clupisoma garua, Clupisoma montana, Semileptus gongota, Acanthocobitis botia and Labeo bata. Some of these fish find entry into the beels during floods, when Indian major carp also enter from neighboring water bodies and culture ponds. As per Molur 
Table 1. List of fishes followed by local names, distribution in northeastern states and national threat categories as per IUCN (Molur \& Walker 1998)

\section{Order: Osteoglossiformes}

Family: Notopteridae

1 Notopterus notopterus (Pallas,1769), Kanduli, Am, Ap, Mg, Mn, Tr (LRnt)

2 Chitala chitala (Hamilton, 1822), Chital, Am, Mg, Mn, $\operatorname{Tr}(\mathrm{EN})$

\section{Order: Clupeiformes}

Family: Clupeidae

3 Gudusia chapra (Hamilton,1822), Karati, Am, Mg, Mn, (LRIc)

\section{Order: Cypriniformes}

Family: Cyprinidae

4 Salmophasia bacaila* (Hamilton,1822), Chelakani, Am, Ap, Mg, Mn, $\operatorname{Tr}(\mathrm{LRlc})$

5 Salmophasia phulo* (Hamilton,1822), Chelakani, Am, Ap, Mg, Mn (NE)

6 Barilius bendelisis (Hamilton,1822), Bariola, Am, Ap, Mg, NI, Mn, Mi, Tr (NE)

7 Chela laubuca (Hamilton,1822),Darikona , Am, Ap, Mg, Mn, Tr (LRnt)

8 Esomus danricus (Hamilton,1822), Dorikona, Am, Ap, Mg, NI, Mn, Tr (LRIc)

9 Danio rerio (Hamilton,1822), Lauputi, Am, Ap, NI, Mi, Tr (LRnt)

10 Rasbora daniconius (Hamilton,1822), Shalynnai, Am, Ap, Mg, Mn, $\operatorname{Tr}(\mathrm{NE})$

11 Amblypharyngodon mola (Hamilton, 1822), Moah, Am, Ap, Mg, Mn, $\operatorname{Tr}(\mathrm{NE})$

12 Puntius chola (Hamilton,1822), Puthi, Am, Ap, Mg, Mn, Tr (VU)

13 Puntius sarana (Hamilton,1822), Cheniputhi, Am, Ap, Mg, Mn, Tr (VU)

14 Puntius conchonius (Hamilton,1822), Puthi, Am, Ap, Mg, Mn, Tr (VU)

15 Puntius sophore (Hamilton,1822), Puthi, Am, Ap, Mg, Mn, Mi, Tr (LRnt)

16 Puntius terio (Hamilton,1822), Puthi, Am, Mg, Mn, Mi, Tr (LRnt)

17 P ticto (Hamilton,1822), Puthi, Am, Ap, Mg, NI, Mn, Mi, Tr (LRnt)

18 Cirrhinus reba (Hamilton,1822), Lachim, Am, Ap, Mg, Mn, Mi, Tr (VU)

19 Catla catla (Hamilton,1822), Bhakua, Am, Mn, Tr (VU)

20 Labeo bata (Hamilton,1822), Bata Am, Ap, Mg, Tr (LRnt)

21 Labeo calbasu (Hamilton,1822), Kaliajora Am, Ap, Mg, Mn, Mi, Tr (LRnt)

22 Labeo gonius (Hamilton,1822), Kurhi Am, Ap, Mg, Tr (LRnt)

23 Labeo rohita (Hamilton, 1822), Rau, Am, Ap, Mg, Tr (LRnt)

\section{Family: Balitoridae}

24 Acanthocobitis botia (Hamilton,1822), Botia, Am, Ap, Mg, Tr (NE)

\section{Family: Cobitidae}

25 Semileptus gongota (Hamilton,1822) Ganga, Am, Ap, Mg, Tr (LRnt)

26 Lepidocephalichthys guntea (Hamilton,1822), Botia, Am, Ap, Mg, NI, Mn, $\mathrm{Mi}, \operatorname{Tr}(\mathrm{NE})$

27 Lepidocephalichthys menoni (Pillai \&Yazdani, 1976), Botia Am (VU)

28 Lepidocephalichthys goalparensis (Pillai \&Yazdani, 1976), Botia, Am (CR)

\section{Order:Siluriformes}

Family: Bagridae

29 Batasio tengana (Hamilton,1822), Batasimas, Am, Ap (NE)

30 Hemibagrus menoda (Hamilton,1822), Gagol, Am (NE)

31 Mystus bleekeri (Day,1822), Singorah, Am, Ap, Mg, NI, Mn, Tr (VU)

32 Mystus cavasius (Hamilton,1822), Golsa, Am, Ap, Mg, Mi, Tr (LRnt)

33 Mystus tengara (Hamilton,1822), Tingara, Am (NE)

34 Mystus vittatus (Bloch,1794), Tengra, Am, Ap, Mg, Tr (VU)

\section{Family: Siluridae}

35 Ompok bimaculatus (Bloch,1794), Bami Am, Ap, Mg, NI, Mn, Tr (EN)

36 O pabda (Hamilton,1822), Pabho, Am, Ap, Mg, Mn, Tr (EN)

37 Wallago attu (Bloch \& Schneider,1794), Borali, Am, Ap, Mg, Tr (LRnt)

\section{Family: Schilbeidae}

38 Ailia coilia (Hamilton,1822), Duikata, Am, Ap, Mg, Tr (VU)

39 Neotropius atherinoides (Bloch,1794), Bordaia, Am, Ap, Mg, NI, Mn, Mi, Tr (EN)

40 Clupisoma garua (Hamilton,1822), Naria, Am, Ap, Mg, Mn, Mi, Tr (VU)

41 Clupisoma montana (Hora,1937), Nagra-bocha Am, Tr (NE)

42 Eutropiichthys vacha (Hamilton,1822), Bacha, Am, Ap, Mg, NI, Mn, Mi, Tr (EN)

\section{Family: Claridae}

43 Clarias batrachus (Linnaeus,1754), Magur, Am, Ap, Mg, NI, Mn, Mi, Tr (VU)

44 Heteropneustes fossilis (Bloch,1794), Singhi, Am, Ap, Mg, NI, Mn, Mi, Tr (VU)

\section{Order: Beloniformes}

Family: Belonidae

45 Xenentodon cancila (Hamilton,1822), Kokila, Am, Ap, Mg, NI, Mn, Mi, Tr (LRnt)
Order: Synbranchiformes

Family: Synbranchidae

46 Monopterus cuchia (Hamilton,1822) Kuchia, Am, Ap, Mg, NI, Mn, Tr (LRnt)

Family: Mastacembelidae

47 Mastacembelus armatus (Lacepede,1800), Ghutum, Am, Ap, Mg, NI, Mn, $\mathrm{Mi}, \operatorname{Tr}(\mathrm{NE})$

Order: Perciformes

Family: Chandidae

48. Chanda nama (Hamilton,1822), Chanda, Am, Ap, Mg, Mn, $\operatorname{Tr}$ (NE)

49 Parambassis ranga (Hamilton,1822), Ranga, Am, Ap, Mg, Mn, Mi, $\operatorname{Tr}(\mathrm{NE})$

Family: Nandidae

50 Badis badis (Hamilton,1822), Bharirtala, Am, Ap, Mg, NI, Mn, Mi, Tr (NE)

51 Nandus nandus (Hamilton,1822), Bhada, Am, Ap, Mg, Mn, Tr (LRnt)

Family: Gobiidae

52 Glossogobius giuris (Hamilton,1822), Balia, Am, Ap, Mg, Mn, Mi, Tr (LRnt)

Family: Anabantidae

53 Anabas testudineus (Bloch,1792), Koi, Am, Ap, Mg, Mn, Tr (VU)

Family: Belontidae

54 Colisa fasciatus (Bloch \&Schneider,1801), Kholisa, Am, Ap, Mg, Mn, Tr (LRnt)

55 C lalia(Hamilton,1822) Kholisa, Am, NI, Mn, (NE)

Family: Channidae

56 Channa marulius (Hamilton,1822), Sal, Am, Ap, Mg, Tr (LRnt)

57 Channa punctatus (Bloch,1793), Goroi, Am, Ap, Mg, NI, Mn, Tr (LRnt)

58 Channa striatus (Bloch,1793), Shol, Am, Ap, Mg, NI, Mn, Tr (LRIc)

Order: Tetraodontiformes

Family: Tetraodontidae

59 Tetraodon cutcutia (Hamilton,1822), Gangatope, Am, Ap, Mg, Mn, Tr (LRnt)

Am - Assam; Ap - Arunachal Pradesh; Mg - Meghalaya; NI - Nagaland; Mn Manipur; Mi - Mizoram; Tr - Tripura; NE - Not Evaluated; LRnt - Lower Risk near threatened; LRIc - Lower Risk least concern; VU - Vulnerable; EN Endangered; CR - Critically endangered.

\& Walker (1998), 13 species are Vulnerable, 5 Endangered and 1 Critically Endangered nationally. Eleven species are not evaluated in the CAMP workshop (Molur \& Walker 1998) have been recorded. Fishing goes on throughout the year in these beels. In the surrounding area paddy fields are present. Runoff water from these fields finds entry into these beels.

\section{References}

ARSAC (1997). Report of Wetlands of Assam: Assam Remote Sensing Application Centre, Guwahati, Assam, India.

Bordoloi, S. \& A. Baishya (2006). Puntius ornatus a new record for the Brahmaputra drainage and the state of Assam. Zoos' Print Journal 21(6): 2292-2294.

Molur, S. \& S. Walker (1998). Fresh water fishes of India. Conservation, Assessment and Management Plan (CAMP) workshop, NBFGR, Lucknow. 22-26 September, 156pp

Das, P. \& S.C. Bordoloi (1997). Fish fauna of a Torrential River (Baisistha) in Kamrup District Assam, India. Journal of Inland Fisheries society of India 29(1): 54-58

Dey, S.C. (1981). Studies on the hydrobiological conditions of some commercially important Beels of Kamrup district of Assam and their bearing on fish production, Final Technical report, N.E.C., $177 \mathrm{pp}$.

Dey, S.C. (1984). Community fishing in Assam and its impact on beel fisheries, Assam Science Society, Conference Proceding 31: 14-15.

Dutta \& Lahon (1987). Prospects and potentialities of beel fishery in Assam. Compendium of Workshop on Development of Beel fishery 
in Assam, 1-7pp.

Fishbase (2009). http:/ / fishbase.org (accessed on April 14, 2009)

Goswami, M.M., T.K. Deka, P.K. Sarma \& M. Kakati (1999). Studies of some wetlands of Assam with reference to the Eutrophication stresses. Journal of Inland Fisheries Society of India 31(2): 39-43.

Jayaram, K.C. (1999). The Freshwater Fishes of Indian Region. Narendra Publishing House, Delhi, 471pp.

Lahon, B. (1983). Limnology and fisheries of some commercial beels of Assam, India, Unpublished Ph.D Thesis, G.U., 349pp.

Sarkar, U.K. \& A.G. Ponniah (2000). Evaluation of North East Indian fishes for their potential as cultivable, Sport and ornamental fishes alongwith their conservation and endemic status. Workshop on North East Indian fish germplasm inventory and conservation, 1-17pp.

Sen, N. (2003). Fish fauna of north east India with special reference to endemic and threatened species, Records of the Zoological Survey of India 101(3\&4): 81-99.
Talwar, P.K. \& A.G. Jhingran (1991). Inland Fishes of India and Adjacent Countries Vol 1 \& 2. Oxford and IBH publishing co. pvt. Ltd., $1158 \mathrm{pp}$

Yadav, Y.S. (1987). Studies on the limnology and productivity of an oxbow lake in Dhubri District of Assam India, Ph. D Thesis, G.U.

Yadav, Y.S. \& M. Choudhury (1981). Katal fishing- a special device for catching fish in beels of Assam. Journal of Inland Fisheries Society of India 13(1): 81-86.

Yadav, Y.S. \& M. Choudhury (1986). Banas fishing in beels of Assam. Journal of the Bombay National History Society 83(2): 452-456.

Yadav, Y.S., R.K. Singh, M. Choudhury \& V. Kolekar (1987). Limnology and productivity of Dighali beel (Assam). Tropical Ecology 28(2): $137-146$. 